\title{
Molecular analysis of oral microflora in patients with primary Sjögren's syndrome by using high-throughput sequencing
}

\author{
Zhifang Zhou ${ }^{1}$, Guanghui Ling ${ }^{1}$, Ning Ding ${ }^{2}$, Zhe Xun ${ }^{1}$, Ce Zhu ${ }^{1}$, Hong Hua ${ }^{3}$, Xiaochi Chen ${ }^{\text {Corresp. }}{ }^{4}$ \\ 1 Department of Preventive Dentistry, Peking University School and Hospital of Stomatology, National Engineering Laboratory for Digital and Material \\ Technology of Stomatology, Beijing Key Laboratory of Digital Stomatology, Beijing, People's Republic of China \\ 2 The 3rd Dental Center, Peking University School and Hospital of Stomatology, National Engineering Laboratory for Digital and Material Technology of \\ Stomatology, Beijing Key Laboratory of Digital Stomatology, Beijing, People's Republic of China \\ 3 Department of Oral Medicine, Peking University School and Hospital of Stomatology, National Engineering Laboratory for Digital and Material Technology \\ of Stomatology, Beijing Key Laboratory of Digital Stomatology, Beijing, People's Republic of China \\ 4 Department of Oral Biology, Peking University School and Hospital of Stomatology, National Engineering Laboratory for Digital and Material Technology \\ of Stomatology, Beijing Key Laboratory of Digital Stomatology, Beijing, People's Republic of China
}

Corresponding Author: Xiaochi Chen

Email address: chenxiaochi@pkuss.bjmu.edu.cn

Background. The objective of this study was to characterize the oral microflora profile of primary Sjögren's syndrome (pSS) patients, thereby revealing the connection between oral bacterial composition and dental caries, and to identify the "core microbiome" in the oral cavities of pSS patients and systemic healthy individuals by using a high-throughput sequencing technique.

Methods. Twenty-two pSS patients and 23 healthy controls were enrolled in this study. Their clinical data and oral rinse samples were collected. The V3-V4 hypervariable regions of the bacterial 16S rRNA gene of samples were amplified and analyzed by highthroughput sequencing on the Illumina Miseq PE300 platform.

Results. Both two groups were age- and sex-matched. There were significantly higher decayed, missing and filled teeth (DMFT) and decayed, missing and filled surfaces (DMFS) in the pSS group than in the control group $(p<0.01)$. Alpha diversity was depleted in pSS patients, compared with healthy controls $(p<0.01)$, while beta diversity between the two groups was not significantly different. Eight discriminative genera (LDA $>4$ ) were found between the two groups in LEfSe (LDA Effect Size) analysis. The relative abundance of Veillonella in pSS patients was fourfold higher, while Actinomyces, Haemophilus, Neisseria, Rothia, Porphyromonas, Peptostreptococcus and Peptostreptococcaceae_XI were significantly lower in pSS patients than in healthy controls. However, the correlation between Veillonella and DMFT/DMFS was not significant $(p>0.05)$. In Venn diagram analysis, nine genera shared by all samples of two groups, which comprised $71.88 \%$ and $67.64 \%$ in pSS patients and controls, respectively.

Discussion. These findings indicate a microbial dysbiosis in pSS patients; notably, Veillonella might be recognized as a biomarker in pSS patients. The core microbiome in 
pSS patients was similar to the systemic healthy population. These provide insight regarding advanced microbial prevention and treatment of severe dental caries in pSS patients. This study also provides basic data regarding microbiology in pSS. 


\section{Molecular Analysis of Oral Microflora in Patients with 2 Primary Sjögren's Syndrome by Using High-throughput 3 Sequencing}

5 Zhifang Zhou ${ }^{1}$, Guanghui Ling ${ }^{1}$, Ning Ding ${ }^{2}$, Zhe Xun ${ }^{1}$, Ce Zhu ${ }^{1}$, Hong Hua ${ }^{3}$, Xiaochi Chen ${ }^{4}$

$6 \quad{ }^{1}$ Department of Preventive Dentistry, Peking University School and Hospital of Stomatology, 7 National Engineering Laboratory for Digital and Material Technology of Stomatology, Beijing

8 Key Laboratory of Digital Stomatology, Beijing, People's Republic of China

92 The 3rd Dental Center, Peking University School and Hospital of Stomatology, National 10 Engineering Laboratory for Digital and Material Technology of Stomatology, Beijing Key

11 Laboratory of Digital Stomatology, Beijing, People's Republic of China

$12{ }^{3}$ Department of Oral Medicine, Peking University School and Hospital of Stomatology, National 13 Engineering Laboratory for Digital and Material Technology of Stomatology, Beijing Key

14 Laboratory of Digital Stomatology, Beijing, People's Republic of China

$15{ }^{4}$ Department of Oral Biology, Peking University School and Hospital of Stomatology, National 16 Engineering Laboratory for Digital and Material Technology of Stomatology, Beijing Key

17 Laboratory of Digital Stomatology, Beijing, People's Republic of China

18 Corresponding Author

19 Xiaochi Chen ${ }^{4}$

2022 Zhongguancun Avenue South, Haidian District, Beijing, 100081, People's Republic of China;

21 Tel: 086-010-82195536

22 E-mail address: chenxiaochi@pkuss.bjmu.edu.cn 


\section{Abstract}

24 Background. The objective of this study was to characterize the oral microflora profile of primary Sjögren's syndrome (pSS) patients, thereby revealing the connection between oral bacterial composition and dental caries, and to identify the "core microbiome" in the oral cavities of pSS patients and systemic healthy individuals by using a high-throughput sequencing

28 technique.

Methods. Twenty-two pSS patients and 23 healthy controls were enrolled in this study. Their clinical data and oral rinse samples were collected. The V3-V4 hypervariable regions of the bacterial 16S rRNA gene of samples were amplified and analyzed by high-throughput sequencing on the Illumina Miseq PE300 platform.

Results. Both two groups were age- and sex-matched. There were significantly higher decayed, missing and filled teeth (DMFT) and decayed, missing and filled surfaces (DMFS) in the pSS group than in the control group $(p<0.01)$. Alpha diversity was depleted in pSS patients, compared with healthy controls $(p<0.01)$, while beta diversity between the two groups was not significantly different. Eight discriminative genera (LDA $>4$ ) were found between the two groups in LEfSe (LDA Effect Size) analysis. The relative abundance of Veillonella in pSS patients was fourfold higher, while Actinomyces, Haemophilus, Neisseria, Rothia,

Porphyromonas, Peptostreptococcus and Peptostreptococcaceae_XI were significantly lower in pSS patients than in healthy controls. However, the correlation between Veillonella and DMFT/DMFS was not significant $(p>0.05)$. In Venn diagram analysis, nine genera shared by all samples of two groups, which comprised $71.88 \%$ and $67.64 \%$ in pSS patients and controls,

44 respectively.

Discussion. These findings indicate a microbial dysbiosis in pSS patients; notably, Veillonella might be recognized as a biomarker in pSS patients. The core microbiome in pSS patients was similar to the systemic healthy population. These provide insight regarding advanced microbial prevention and treatment of severe dental caries in pSS patients. This study also provides basic data regarding microbiology in pSS.

Keywords Primary Sjögren's syndrome; Oral microflora; Core microbiome; Highthroughput sequencing; Dental caries 


\section{1. Introduction}

53

54

55

56

57

58

59

60

61

62

63

64

65

66

67

68

69

70

71

72

73

74

75

76

77

78

79

80

81

82

83

84

85

86

87

88

89

90

91

92

93

94

As one of the most common chronic autoimmune diseases, Sjögren's syndrome (SS) destroys salivary gland tissue, which leads to severe xerostomia (dry mouth) and impairs oral health. The SS prevalence rate is $0.61 \%$, occurring 10 times more frequently in women than in men (female/male ratio in prevalence data was 10.72); it occurs most commonly in middle-aged women between 40-60 years of age (Qin et al., 2015). Primary Sjögren's syndrome (pSS) is marked by a variable degree of activated $\mathrm{CD}^{+} \mathrm{T}$ cell and $\mathrm{B}$ cell infiltration (Christodoulou, Kapsogeorgou \& Moutsopoulos, 2010) of the secretory glands. However, secondary Sjögren's syndrome (sSS) is associated with other connective tissue diseases, such as systemic lupus erythematosus (SLE) and rheumatoid arthritis (RA) (Vitali et al., 2002). Xerostomia hinders eating, speaking, and swallowing (all of which largely compromise quality of life for SS patients), and causes rampant caries that always occur in individuals with low saliva flow (Scully, 1986; Gravenmade \& Vissink, 1992; Lin et al., 2010).

Researchers have performed several experiments to study oral bacteria in pSS patients. Almståhl et al. cultured and counted the Lactobacillus species collected from the supragingival plaque in subjects with hyposalivation (ten subjects with radiation-induced hyposalivation and ten with pSS) and identified them by PCR and restriction fragment length polymorphism (RFLP), and they reported large intra- and inter-individual variations in number and frequencies of Lactobacilli (Almståhl et al., 2010). Leung et al. investigated the level of oral micro-organisms in SS patients by using a selective culture technique. They found that the level of Lactobacillus acidophilus in stimulated whole saliva $(p=0.012)$ and noncaries associated supra-gingival plaque $(p<0.0001)$ were significantly higher in $\mathrm{pSS}$ patients than in healthy controls and sSS individuals (Leung, Leung \& McMillan, 2007).

Although there have been several studies conducted on the oral micro-organisms of pSS patients, the experimental methods previously used were mostly based on bacterial culture, which is limited to several cariogenic bacteria already known to us. Moreover, traditional methods of culture can only recognize approximately 280 species of oral microbes (Dewhirst et al., 2010), while there are more than 700 species in the human oral cavity (Aas et al., 2005). Fortunately, a more advanced biotechnique, high-throughput sequencing, has emerged recently, allowing researchers to detect all oral microbes among samples in a single experiment and reveal deeper insights regarding the oral microflora. Siddiqui et al. reported a twofold increase in the relative abundance of Streptococcus and Veillonella in pSS patients, indicating that microbial dysbiosis was a key characteristic of pSS whole saliva; this dysbiosis was found to occur independent of hyposalivation, after analysis of whole unstimulated saliva of pSS patients and healthy controls, both with normal salivation, by high-throughput sequencing (Siddiqui et al., 2016). With a similar biotechnique, a preliminary study regarding the buccal mucosa microflora of pSS patients found that pSS patients carried a different and less diverse microbial community than that of healthy subjects (Li et al., 2016). The pSS group had a greater relative abundance of Leucobacter, Delftia, Pseudochrobactrum, Ralstonia and Mitsuaria but exhibited reduced abundance of Haemophilus, Neisseria, Comamona, Granulicatella and Limnohabitans, compared with the control group $(p<0.05)$. de Paiva et al. studied the tongue microbiome of SS patients and found a significant decrease in diversity $(p<0.05)$ and differences in the composition of the microflora in SS patients, compared with their healthy controls (de Paiva et 
al., 2016). An increased relative abundance of Streptococcus and a decreased relative abundance of Leptotrichia and Fusobacterium in the SS group were observed, compared with controls ( $p<$ 0.05 for all). Though there have been several studies exploring the oral microflora of pSS or SS patients via high-throughput sequencing, the discriminative genera between patient and control groups among the three studies were diverse; notably, none of them discussed the relationship between dental caries and oral microbiota. Thus, the aim of this study was to characterize the oral microflora profile of pSS patients, to identify the "core microbiome" in the oral cavity of pSS patients, and to reveal the connection between oral bacterial composition and dental caries via high-throughput sequencing technique and provide basic data for the oral microbiology of pSS.

\section{Materials \& Methods}

\subsection{Subjects}

According to the significantly higher SS prevalence rate in women (female/male ratio in prevalence data was 10.72), all patients in our study are female. Twenty-two patients from the Department of Oral Medicine of Peking University School and Hospital of Stomatology were enrolled. The patients were diagnosed with pSS according to the revised international classification criteria (Vitali et al., 2002). The control group consisted of 23 age-matched females from a community in Beijing (The Xili community of Xinjiekou Street, Xicheng District, Beijing). All individuals in the control group were systemic healthy and had no xerostomia symptoms. All participants in our study were enrolled who reported no diabetes mellitus, had not received radiotherapy, had not taken drugs with side effects of dry mouth for 3 months and had no oral mucosal lesion during sampling. They were examined for DMFT and DMFS. Clinical examination and sample collection were performed by a single experienced dentist. The examiner was previously trained and passed the standard consistency test (Kappa $>0.8)$. All subjects signed written informed consent, and the study passed the ethical review of the Institutional Review Board of Peking University School and Hospital of Stomatology, \#IRB00001052-12025.

\subsection{Microbial sampling and DNA isolation}

Tooth-brushing was not allowed from the night before the appointment until the end of sampling. All subjects were instructed to refrain from eating and drinking for at least 2 hours prior to the clinical examination. The examination was performed between 9 A.M. and 11 A.M. The rinse samples were collected into sterile graduated test-tubes after the subjects rinsed their mouths thoroughly for $1 \mathrm{~min}$ with $10 \mathrm{ml}$ physiological saline. Samples were immediately transported on ice to the laboratory and centrifuged at $9000 \mathrm{~g}$ for $10 \mathrm{~min}$. The supernatant was discarded and the precipitate was stored at $4^{\circ} \mathrm{C}$. Within $2 \mathrm{~h}$, the samples were processed following the method of Gao (Gao et al., 2013). The precipitate was incubated for $1 \mathrm{~h}$ at $58^{\circ} \mathrm{C}$ with $1 \mathrm{ml}$ of lysis buffer ( $10 \%$ sodium dodecyl sulfate (SDS) and $0.2 \mathrm{mg} / \mathrm{ml}$ proteinase $\mathrm{K}$ in $25 \mathrm{mM}$ Tris- $\mathrm{HCl}, \mathrm{pH} 8$ ), followed by incubation at $80^{\circ} \mathrm{C}$ for $10 \mathrm{~min}$ to denature the proteinase $\mathrm{K}$. DNA was purified from the lysate by repeated phenol-chloroform-isoamyl alcohol extraction, precipitated with sodium 
136

137

138

139

140

141

142

143

144

145

146

147

148

149

150

151

152

153

154

155

156

157

158

159

160

161

162

163

164

165

166

167

168

169

170

171

172

173

174

175

acetate and ethanol, and dissolved in $100 \mu \mathrm{l}$ sterile Milli-Q water. The final DNA concentration was determined by NanoPhotometer ${ }^{\mathrm{TM}}$ Pearl ultramicro ultraviolet spectrophotometer (Implen, Munich, Germany). The DNA quality was checked by $1 \%$ agarose gel electrophoresis. A negative control only with buffer was involved during DNA isolation and quantification. The resulting DNA was stored at $-80^{\circ} \mathrm{C}$ before further analysis.

\subsection{High-throughput sequencing}

The samples were conveyed to MyGenostics lnc. (Beijing, China) for high-throughput sequencing. The V3-V4 hypervariable regions of the bacterial 16S rRNA (16S ribosomal RNA) gene were amplified with primers 338F (5'- ACTCCTACGGGAGGCAGCAG-3') and 806R (5'-GGACTACHVGGGTWTCTAAT-3') (Lu et al., 2016) by PCR (GeneAmp 9700, ABI, USA), followed by the electrophoresis for quality inspection. Once again, a negative control only with buffer was enrolled during DNA amplification and electrophoresis. The amplified products were sequenced on the Illumina Miseq PE300 platform (Illumina, San Diego, USA).

\subsection{Data analysis and statistical methods}

The demographic and clinical data were collected and processed via SPSS 20.0. Two-tailed independent-samples t-tests, chi-square test and Spearman's rank correlation were used to analyze the data we obtained.

The raw reads obtained by high-throughput sequencing (deposited in the NCBI Sequence Read Archive with accession SRP133569) were processed via Mothur 1.38.1 (Schloss et al., 2009) and QIIME 1.9.1 (Caporaso et al., 2010). The reads were underwent paired-end reads merging using Mothur. The de-novo strategy for chimera filtering was employed using VSEARCH (v2.0.2). The quality filtering strategy was performed by truncating those reads shorter than $200 \mathrm{bp}$ in length, those with quality score $<20$, mononucleotide repeats and homopolymers $>10 \mathrm{bp}$ using QIIME. The trimmed and optimized sequences were clustered for operational taxonomy unit (OTU) analysis at a 97\% similarity threshold using the QIIME implementation of UCLUST (Edgar, 2010). The representative sequences from each OTU were aligned in the Human Oral Microbiome Database (HOMD Release 14.5) (Chen et al., 2010) by QIIME.

Bioinformatics analyses, such as alpha diversity (Ace, Chao1, Pielou, Shannon and Simpson indices), beta diversity (PCoA based on weighted Unifrac matrix) were performed in accordance with the OTU table in QIIME. The data of alpha diversity were evaluated via independentsamples t-tests or nonparametric tests in terms of normality of distribution and visualized by ggplot2 package in R language (v2.2.0). The significance of PCoA was accessed by PERMANOVA test and visualized by ggplot 2 package in $\mathrm{R}$ language. LEfSe analysis was performed on the online Galaxy workflow framework (http://huttenhower.sph.harvard.edu/galaxy/) (Segata et al., 2011); the threshold on the logarithmic linear discriminant analysis (LDA) score was set to 4.0. Heatmap analysis was performed using pheatmap package in $\mathrm{R}$ language (v1.0.10). A $p$ less than 0.05 was considered statistically significant. 
176

177 3. Results

\section{$178 \quad$ 3.1 General outline}

179 To characterize the oral microflora profile of pSS patients, and explore the relationship between

180 pSS and caries, we analyzed the sequences of 16S rRNA gene of rinse samples from 45 subjects

181 (22 samples from pSS patients and 23 samples from healthy controls). The average age in both

182 groups was not significantly different $(p=0.08)$. The DMFT and DMFS of patients were

183 significantly higher than those of the control group $(p<0.01)$. The prevalence of caries in

184 patients was $95.45 \%$, whereas it was $69.57 \%$ in the control group, but the difference was not

185 significant $(p=0.06)$. These data are presented in Table 1.

186 After sample detection, all 45 samples were qualified for high-throughput sequencing, including

18722 rinse samples of pSS patients (designated as "PR") and 23 rinse samples of healthy subjects

188 (designated as "CR"). We obtained 2579816 raw reads via sequencing. A total of 2335289 reads

189 were involved after quality filtration, with an average of 51895 effective reads per sample. A

190 total of 16502 operational taxonomic units (OTUs) were observed across all samples and ranged

191 from 357 to 1852 per sample.

192

193 3.2 Global taxonomic features and microbial diversity characteristics in pSS 194 patients and healthy controls

195 The results of classification and annotation at the phylum and genus levels (only 62 genera with relative abundance $>1 \%$ are listed) for all samples are shown in Fig. S1. A total of 11 phyla were found across all samples. The dominant phyla included Firmicutes, Actinobacteria, Proteobacteria, Bacteroidetes and Fusobacteria, which comprised $99.33 \%$ and $98.82 \%$ in pSS patients and controls, respectively. A total of 158 genera were found across samples.

200 Streptococcus, Actinomyces, Veillonella, Rothia, Prevotella, Neisseria, Haemophilus, Granulicatella, Fusobacterium, Peptostreptococcus, Gemella, Corynebacterium, Klebsiella, Enterobacter and Ralstonia were the top 15 most abundant genera, which comprised $87.16 \%$ and $84.11 \%$ in pSS patients and healthy controls, respectively.

204

205

206

207

208

209

210

211

212

213

214
To access the information about the oral microbial communities of pSS patients and healthy controls, we performed the heatmap analysis (Fig. 1). All subjects were divided into two clusters (Cluster 1 and Cluster 2) according to the similarity of samples. All the healthy controls except one were distributed in Cluster 2. Though pSS patients were relatively scattered, Cluster 1 was almost constructed by them (Fig. 1).

Alpha diversity indices are used to demonstrate the microbial community richness, evenness, and species diversity in sites or habitats at a local scale. Bacterial richness between the two groups was not significantly different, according to Ace and Chao1 indices (Fig. 2A and 2B). However, oral bacterial community evenness and diversity in the pSS group were significantly lower than that in the control group, according to the Pielou, Shannon and Simpson indices $(p<0.01)$ (Fig. $2 \mathrm{C}, 2 \mathrm{D}$ and $2 \mathrm{E})$. 
215 Beta diversity analysis, such as PCoA (principal co-ordinates analysis) via weighted UniFrac

216 distance metrix, was executed to compare the overall bacterial community composition of the

217 two groups. No significant segregation was found between patients and healthy controls

218 (PERMANOVA, $p=0.63$, Fig. 2F). However, the dots of control group were more concentrated

219 than pSS group as illustrated in the figure.

220

\section{$221 \quad 3.3$ Discriminative taxa of pSS patients and healthy controls}

222

223

224

225

226

227

228

229

230

231

232

233

234

235

236

237

238

239

240

241

242

243

244

245

246

247

248

249

250

251

252

253

254

255

256

The LEfSe (LDA Effect Size) analysis was performed for the exploration of relative taxa abundance, characterized by significant differences between the two groups (i.e., biomarkers). These taxa likely play a crucial role in their local microenvironment; they are listed in Fig. 3. At the genus level, the relative abundance of Veillonella in patients (12.22\%) was fourfold higher than in the healthy controls (3.21\%). However, a relatively higher abundance of Actinomyces, Haemophilus, Neisseria, Rothia, Porphyromonas, Peptostreptococcus and

Peptostreptococcaceae_XI was observed in healthy controls, compared with pSS patients. Notably, the well-known cariogenic bacteria such as Streptococcus and Lactobacillus were found no significant difference between two groups. We performed Spearman's rank correlation to explore the relationship between the relative abundance of Veillonella and the severity of caries (Fig. S2), but no great significance was found.

\subsection{Core microbiome of pSS patients and healthy controls}

The core microbiome is defined as the taxa shared across all samples in a certain group or among groups; it is illustrated by using a Venn diagram (Fig. 4). A total of 158 genera were identified by OTU annotation; 149 genera belonged to pSS patients and 136 to healthy controls. The overlap region A represents the microbiota shared in all samples among patient and control groups, including nine genera: Leptotrichia, Rothia, Actinomyces, Granulicatella, Porphyromonas, Prevotella, Veillonella, Fusobacterium, Streptococcus. There was no other genus shared among pSS patients but an additional 27 genera (region B) were shared among healthy controls, including Stomatobaculum, Dialister, Olsenella, Capnocytophaga, Peptococcus, Propionibacterium, Bergeyella, Megasphaera, Selenomonas, Lautropia, Lachnoanaerobaculum, Parvimonas, Catonella, Alloprevotella, Campylobacter, Solobacterium, Oribacterium, Actinobaculum, Atopobium, Neisseria, Corynebacterium, Gemella, Haemophilus, Peptostreptococcus, TM7_[G-1], Peptostreptococcaceae_[XI][G-1], Unclassified_Lachnospiraceae_[XIV]. Thirty-six genera (region A and B) were the core microbiome of healthy controls. Twenty-two genera in region D of PR (Kocuria, Mycobacterium, Flavitalea, Escherichia, Cupriavidus, Pedobacter, Anaerococcus, Cronobacter, Ralstonia, Stenotrophomonas, Burkholderia, Rhodocyclus, Unclassified_Actinobacteria, Unclassified_Alcaligenaceae, Unclassified_Lactobacillales, Clostridiales_[F-3][G-1], Unclassified_Rhizobiaceae, Unclassified_Sphingomonadaceae, Unclassified_Carnobacteriaceae, Unclassified_Alphaproteobacteria, Unclassified_Corynebacteriales, Unclassified_Betaproteobacteria) represented the unique taxa in pSS patients, while nine genera in region D of CR (Eggerthella, Peptoniphilus, Sneathia, Ruminococcaceae_[G-3], Syntrophomonadaceae_[VIII][G-1], GN02_[G-1], 
257

258

259

260

261

262

263

264

265

266

267

268

269

270

271

272

273

274

275

276

277

278

279

280

281

282

283

284

285

286

287

288

289

290

291

292

293

294

295

296

297

Unclassified_Bifidobacteriaceae, Clostridiales_[F-1][G-2],TM7_[G-6]) represented the unique taxa in healthy controls. The relative abundances of the core microbiome and unique genera are shown in Table 2.

\section{Discussion}

By sequencing and analyzing the 16S rRNA gene of oral microflora of pSS patients and healthy controls, we identified the significant differences in alpha diversity of oral microbial community and the relative abundance of Veillonella between pSS patients and healthy controls, which revealed a dysbiosis in oral microflora of pSS patients and characterized the Veillonella may acting as an oral biomarker of pSS.

The rinse sampling method was used in our study. In the context of low salivary secretion in pSS patients and sampling standardization, the rinse technique is more accessible than stimulated saliva and plaque sampling and is capable of assessing oral microbiota structure. Several studies have used this method to investigate the oral microbiota. For instance, Almståhl et al. compared the oral microbiota associated with hyposalivation of different origins by analyzing with rinse samples and found the number of $S$. mutans in pSS patients was significantly higher than in the control group with normal salivary secretion $(p=0.004)$ (Almståhl et al., 2003). Other studies have suggested that the rinse technique is reliable for measuring oral microflora as well (Samaranayake et al., 1986; Fure \& Krasse, 1988).

More bacteria taxa, even nonculturable ones, can be detected, and the diversity and composition of oral microflora can be studied, by using high-throughput sequencing, in contrast to counting the numbers of selected bacteria by traditional culture methods. Significant differences in oral microbial ecology were present between pSS patients and their healthy counterparts in our study.

The pSS population is known to exhibit higher DMFT and DMFS. A comprehensive study assessing the oral health status of pSS patients reported a higher DMFT among pSS patients compared with healthy controls, regardless of age $(p<0.05)$ (Christensen et al., 2001). In addition, a study noted that DMFS in pSS patients was higher than in healthy controls $(p=0.01)$ and DMFS correlated positively with oral dryness $\left(r_{s}=0.53, p<0.05\right)$ (Pedersen, Bardow \& Nauntofte, 2005). Data from relevant studies also showed that pSS patients had higher DMFT than their healthy counterparts ( $p<0.05$ of both) (Pozharitskaia \& Morozova, 1988; Leung, Leung \& McMillan, 2007). Besides the decrease of salivary flow which impaired the functions of oral cleaning and buffering capacity, the change in oral bacteria was another factor to aggravate the development of dental caries. Therefore, the relationship between pSS and cariogenic bacteria was investigated in several studies. An increased level of S. mutans and/or Lactobacillus spp. was observed in the oral microflora of patients with pSS, compared with healthy subjects, by selective culture (Almståhl, Wikström \& Kroneld, 2001; Almståhl et al., 2003; Leung, Leung \& McMillan, 2007). However, no significant differences in Streptococcus and Lactobacillus were observed between patients and healthy controls in this study. In general, there are possible several reasons for this result. Firstly, though the DMFT and DMFS in healthy controls were significantly lower than in pSS patients $(p<0.01)$, the prevalence of dental caries was high and had no great significance compared with pSS group $(p=0.06)$. Therefore, their 
298

299

300

301

302

303

304

305

306

307

308

309

310

311

312

313

314

315

316

317

318

319

320

321

322

323

324

325

326

327

328

329

330

331

332

333

334

335

336

337

338

339

340

341

342

oral microflora composition may be closer to the population with high risk of caries which indicated by the increased level of Lactobacillus and cariogenic streptococci, rather than the population with low risk of caries $(p<0.05)$ (Arino et al., 2015); this suggests that these bacteria may be incapable of serving as specific biomarkers of pSS patients with a high caries risk. Hence, no obvious differences in the relative abundance of specific cariogenic micro-organisms were observed between the two groups. Secondly, defining the organisms at the genus level, rather than at the species level, is a limitation of 16S rRNA high-throughput sequencing (Rosselló-Mora \& Amann, 2001). Importantly, in certain genera, the variation of pathogenicity among species can be remarkable. For instance, over 50 species are recognized in Streptococcus whereas $S$. mutans is the most prominent cariogenic species among streptococcal population (according to a large body of existing evidence). Other streptococci, known as the "non-mutans streptococci" (Kawamura et al., 1995), are predominant in integral tooth surfaces. Even Streptococcus salivarius, an oral cavity-based commensal species in this genus, is regarded as a safe and efficacious probiotic capable of maintaining healthy oral microbiota and promoting oral health (Wescombe et al., 2012). In consideration of these factors, the relative abundance of cariogenic bacteria in various degrees between the two groups may be perceived if the data is accurate at the species level.

The relative abundance of Veillonella in the pSS group (12.22\%) was significantly higher than in the control group (3.21\%) (LDA score $>4$ ); this result is consistent with several recent studies (Hayashi et al., 2014; Zhang et al., 2015; Siddiqui et al., 2016). Veillonella is a Gram-negative anaerobic cocci; its salient physiological characteristic is its lactate-fermenting ability (Delwiche, Pestka \& Tortorello, 1985), which enables production of propionic and acetic acids, carbon dioxide, and hydrogen (Rogosa, 1964). Although the increase of lactate acid led to a raise of Veillonella and a decrease in lactic acid, it would eventually hoist the amount of total acid; moreover, there was a symbiotic relationship between S. mutans and Veillonella (Noorda et al., 1988). Another study also showed that the presence of Veillonella mainly increased biofilm formation by Streptococcus species (Mashima \& Nakazawa, 2014). A metagenomic analysis of the bacteria in dental cavities showed that Veillonella was one of the most common genera in cavities (Belda-Ferre et al., 2012), that was in agreement with a molecular study which also considered Veillonella as one of the potential cariogenic bacteria (Aas et al., 2008). Notwithstanding, the correlation between Veillonella and DMFT/DMFS was not distinct in this study $(p>0.05)$. Therefore, we assumed that the increased level of Veillonella might be potentially affected by the disease condition. However, further more rigorous and precise experiments are needed to validate the Veillonella acting as a biomarker of pSS in the future.

The relative abundances of Actinomyces, Rothia, Peptostreptococcus, Haemophilus, Neisseria, Porphyromonas and Peptostreptococcaceae_XI were reduced in pSS patients in the present study. Actinomyces, together with the non-mutans streptococci, predominates on sound enamel tooth surfaces (Ximénez-Fyvie, Haffajee \& Socransky, 2000). With the development of dental caries, the microflora switches from dominance by non-mutans streptococci and Actinomyces to dominance by $S$. mutans and other non-mutans bacteria, including lactobacilli and Bifidobacterium (Takahashi \& Nyvad, 2011). Similiar to Actinomyces, but lesser in abundance, Rothia is a frequently observed genus in the initial dental plaque community (Diaz et al., 2006) and one of the predominant bacteria in the oral cavity of healthy individuals (Keijser et al., 2008; Bik et al., 2010). Therefore, it is not surprising that Actinomyces and Rothia were richer in healthy controls than in pSS patients in our findings. 
$343 \mathrm{Li}$ et al. investigated the buccal mucosa microbiota in pSS patients; they reported a lower relative 344 abundance of Haemophilus and Neisseria in patients $(p<0.05)$ (Li et al., 2016). In addition,

345 Neisseria exhibited a reduced abundance in subjects with hyposalivation, compared with subjects 346 with normo-salivation, as observed by a team of Japanese scientists (Hayashi et al., 2014). In an

347 analysis of salivary microbiota in individuals with different levels of caries experience, a twofold higher relative abundance of Neisseria and Haemophilus was recorded in the group with low caries experience than in the group with high caries experience (Belstrom et al., 2017). Most species in Neisseria and Haemophilus genera only mildly ferment sugar (Belstrom et al., 2017) and exhibit a low acid resistance. As a non-caries-associated species, for instance, Neisseria subflava cannot be cultured in the environment where $\mathrm{pH}$ is lower than 4.5 (Bradshaw \& Marsh, 1998). Thus, it is reasonable to speculate that the reduction of non-caries-associated taxa is due to a lower $\mathrm{pH}$ environment in $\mathrm{pSS}$ patients, which may result from damage to the buffering function of salivary glands. Though we have not measured salivary or plaque $\mathrm{pH}$ in this study. given these circumstances, it is evident that the pSS patients possess an exceptional bacterial composition and a higher level of dental caries than healthy controls.

There was a significantly lower microbial community diversity in the pSS group than in the control group, consistent with previous reports (de Paiva et al., 2016; Li et al., 2016; Siddiqui et al., 2016). The similarity in richness of the two groups can be attributed to the lower evenness in degree in the pSS group; the results of Pielou analysis confirmed that hypothesis. Despite the disease itself, the huge variation in saliva flow is one of the explanations of the significantly reduced microbial diversity in pSS patients. The saliva maintains a balanced homoeostasis between the highly diverse oral microbiome and oral health status by various mechanisms, such as MUC5B (one of the mucoprotein that is encoded by the MUC5B gene), lysozyme, histatins, $\beta$-defensins and the human cathelicidin LL37 (van't Hof et al., 2014). Therefore, decreased saliva flow rate and changed composition that disrupt the biocoenosis of the oral cavity of the pSS patients are crucial factors causing reduced diversity of the oral microflora in pSS patients. Meanwhile, autoimmune diseases and medication can also influence the oral microbiota, and some researchers still suspect a more complex mechanism (Szymula et al., 2014; Zhang et al., 2015; Li et al., 2016; Siddiqui et al., 2016; van der Meulen et al., 2016). Li et al. analyzed the impaction of medication on the buccal microflora of pSS patients and suggested that Streptococcus and Lactobacillus were obviously affected by treatment with prednisone acetas (Li et al., 2016). Another study described a pronounced increase of Streptococcus and Veillonella in pSS patients with normal salivation, which emphasizes that the disturbance in the biocoenosis of pSS patients can occur independent of hyposalivation (Siddiqui et al., 2016). In a study of dental and salivary micro-organisms of individuals with rheumatoid arthritis (RA) launched by Zhang et al., reductions of Haemophilus spp., Porphyromonas gingivalis and Rothia aeria were observed in the oral cavities of RA patients (Zhang et al., 2015). SSA (Sjögren's syndrome Antigen A)/Ro60 is a major autoantigen in SS and SLE (Schulte-Pelkum, Fritzler \& Mahler, 2009). A recent study with laboratory research revealed that the von Willebrand factor type A (vWFA) domain protein produced by Capnocytophaga ochracea was the most potent activator of SSA; further, SSA/Ro60-reactive T cells were activated by recombinant vWFA protein (Szymula et al., 2014). These results demonstrate that the autoimmune responses to the normal human microbes and opportunistic pathogens might be the potential trigger of initial autoimmunity in SS and SLE. These observations emphasize that there is change in the oral microbial diversity of patients affected by the disease itself and by medication; inversely, the human microbiota conditions the individual's autoimmunity, which has a strong relationship with 
389 hereditary susceptibility. Given the complex and unclear interaction between the oral microbiota and autoimmune diseases, this merits further exploration.

391

392

393

394

395

396

397

398

399

400

401

402

403

404

405

406

407

408

409

410

411

412

413

414

415

416

417

418

419

420

421

422

423

424

425

426

427

428

429

430

431
In contrast to the recent study (de Paiva et al., 2016), there was no clear difference in the beta diversity of the two groups $(p=0.63)$. Considering that de Paiva et al. reported a significantly lower beta diversity in SS group than in control group $(p=0.002)$ when sequenced the tongue microbiome from ten SS patients and eleven healthy controls, we speculated that the design of subjects rather than the sample size attributed to this result: the earlier study reckoned without the impact of dental caries, whereas we took that factor into account.

A total of 149 genera in pSS patients and 136 genera in healthy controls were found and 9 genera overlapped in Venn diagram. Shared genera are common in the human oral cavity, comprising $71.88 \%$ and $67.64 \%$ of the relative abundance of microbiota of patients and controls, respectively. Aas et al. amplified and sequenced the bacterial 16S rDNA from nine oral sites (including tongue dorsum, buccal epithelium, supragingival and subgingival plaque and so forth) of five healthy adults (Aas et al., 2005). They found that the genera Gemella, Granulicatella, Streptococcus, and Veillonella were present in all sites. Xiao et al. sequenced the V1-V3 hypervariable region of bacterial $16 \mathrm{~S}$ rDNA of supra-gingival plaque of 160 subjects with various levels of dental caries; 99 shared genera in all samples were considered as the core plaque microbiome in those adults. Six genera with the highest relative abundance were Capnocytophaga (17.8\%), Prevotella (13.5\%), Actinomyces (13.0\%), Corynebacterium (8.9\%), Streptococcus (6.6\%) and Neisseria (6.4\%) (Xiao et al., 2016). To define the healthy core microbiome of the oral microbial community, Zaura et al. sequenced the microflora of five intraoral niches in three healthy adults; 387 OTUs were present in all samples (Zaura et al., 2009). Among these, the abundant taxa (relative abundance more than $0.5 \%$ ) belonged to Streptococcus, Corynebacterium, Neisseria, Rothia, Veillonellaceae, Actinomyces, Granulicatella, Porphyromonas and Fusobacterium. Therefore, nine genera, which were shared in two groups in the present study, are considered as part of the core microbiome of human oral cavity.

The core microbiome of healthy controls consisted of 36 genera, sharing $95.90 \%$ of the abundance. Compared with nine shared genera of patients, the oral microflora of the controls was less variable; the structure of the bacterial community of samples in the control group was more similar to each other for almost all of them were assembled in the Cluster 2 (Fig. 1); that was in general agreement with our results regarding beta diversity, as well as other studies (Zaura et al., 2009; Yang et al., 2012; Xiao et al., 2016). These findings add weight to the hypothesis of the core microbiome in a healthy population. The number of core taxa in patients (nine genera, $71.88 \%$ ) was less than in healthy controls, implying microbial dysbiosis in the oral cavity of patients.

Twenty-two unique genera were detected in the rinse samples of pSS patients; most of the species, such as Kocuria rosea, Cupriavidus gilardii and Anaerococcus prevotii, are opportunistic pathogens that can cause infectious processes (Savini et al., 2010; Chiu \& Wang, 2013; Tennert et al., 2014; Dotis et al., 2015; Du et al., 2015; Zhang et al., 2017), particularly in immunocompromised hosts. Though the pathogenicity of such species is known to us to some extent, the relevance to pSS and dental caries remains undetermined. Nine unique genera appeared in controls, which comprising only $0.05 \%$ of known microbes. The poor acid resistance 
432 and/or environmental sensitivity of these taxa might be the causative factors of deterioration in

433 oral cavities with low saliva flow rates and the severe level of caries in pSS patients.

434

435

436

437

438

439

440

441

442

443

444

445

446

447

448

449

450

451

452

453

454

455

456

457

458

459

460

461

462

463

464

465

466

467

468

469

470

471

\section{Conclusions}

In conclusion, we observed a clear oral microbial dysbiosis among severe dental caries of pSS patients and Veillonella may serve as a biomarker in pSS individuals. The core microbiome of pSS patients was analogous to systemic healthy populations. The results suggest that targeted clinical methods against oral microbes should be considered to prevent the development of severe dental caries in pSS patients.

\section{Acknowledgements}

We appreciate the technical support from MyGenostics lnc. (Beijing, China).

\section{References}

Aas JA, Paster BJ, Stokes LN, Olsen I , Dewhirst FE. 2005. Defining the normal bacterial flora of the oral cavity. Journal of Clinical Microbiology 43(11):5721-5732 DOI:10.1128/JCM.43.11.5721-5732.2005

Aas JA, Griffen AL, Dardis SR, Lee AM, Olsen I, Dewhirst FE, Leys EJ, Paster BJ. 2008. Bacteria of dental caries in primary and permanent teeth in children and young adults. Journal of Clinical Microbiology 46(4):1407-17. DOI:10.1128/JCM.01410-07

Almståhl A, Wikström M , Kroneld U. 2001. Microflora in oral ecosystems in primary Sjögren's syndrome. Journal of Rheumatology 28(5):1007-1013

Almstahl A, Carlen A, Eliasson L, Lingstrom P. 2010. Lactobacillus species in supragingival plaque in subjects with hyposalivation. Archives of Oral Biology 55(3): 255-259

DOI:10.1016/j.archoralbio.2010.01.007

Arino M, Ataru I, Fujiki S, Sugiyama S, Hayashi M. 2015. Multicenter study on caries risk assessment in Japanese adult patients. Journal of Dentistry 43(10):1223-1228 DOI:10.1016/j.jdent.2015.07.010

Belda-Ferre P, Alcaraz LD, Cabrera-Rubio R, Romero H, Simón-Soro A, Pignatelli M, Mira A. 2012. The oral metagenome in health and disease. The ISME Journal 6(1):46-56. DOI:10.1038/ismej.2011.85

Belstrom D, Holmstrup P, Fiehn NE, Kirkby N, Kokaras A, Paster BJ , Bardow A. 2017. Salivary microbiota in individuals with different levels of caries experience. Journal of Oral Microbiology 9(1):1270614 DOI:10.1080/20002297.2016.1270614

Bik EM, Long CD, Armitage GC, Loomer P, Emerson J, Mongodin EF, Nelson KE, Gill SR, Fraser-Liggett CM , Relman DA. 2010. Bacterial diversity in the oral cavity of 10 healthy individuals. The ISME Journal 4(8):962-974 DOI:10.1038/ismej.2010.30

Bradshaw DJ , Marsh PD. 1998. Analysis of pH-driven disruption of oral microbial communities in vitro. Caries Research 32:452-462 DOI:10.1159/000016487 Caporaso JG, Kuczynski J, Stombaugh J, Bittinger K, Bushman FD, Costello EK, Fierer N, Pena AG, Goodrich JK, Gordon JI, Huttley GA, Kelley ST, Knights D, Koenig JE, Ley RE, Lozupone CA, McDonald D, Muegge BD, Pirrung M, Reeder J, Sevinsky JR, Turnbaugh PJ, Walters WA, Widmann J, Yatsunenko T, Zaneveld J, Knight R. 2010. 
472 QIIME allows analysis of high-throughput community sequencing data. Nature Methods

473 7(5):335-336 DOI:10.1038/nmeth.f.303

474 Chen T, Yu WH, Izard J, Baranova OV, Lakshmanan A, Dewhirst FE. 2010. The Human

475 Oral Microbiome Database: a web accessible resource for investigating oral microbe taxonomic

476 and genomic information. Database(Oxford): The Journal of Biological Databases and Curation

477 2010:baq013 DOI:10.1093/database/baq013

478 Chiu LQ, Wang W. 2013. A case of unusual Gram-negative bacilli septic arthritis in an

479 immunocompetent patient. Singapore Medical Journal 54(8):e164-e168

480 DOI:10.11622/smedj.2013162

481 Christensen LB, Petersen PE, Thorn JJ , Schiødt M. 2001. Dental caries and dental health

482 behavior of patients with primary Sjögren syndrome. Acta Odontologica Scandinavica

483 59(3):116-120

484 Christodoulou MI, Kapsogeorgou EK, Moutsopoulos HM. 2010. Characteristics of the minor

485 salivary gland infiltrates in Sjögren's syndrome. Journal of Autoimmunity 34(4):400-407

486 DOI:10.1016/j.jaut.2009.10.004

487 de Paiva CS, Jones DB, Stern ME, Bian F, Moore QL, Corbiere S, Streckfus CF,

488 Hutchinson DS, Ajami NJ, Petrosino JF , Pflugfelder SC. 2016. Altered Mucosal Microbiome

489 Diversity and Disease Severity in Sjögren Syndrome. Scientific Reports 6:23561

490 DOI:10.1038/srep23561

491 Delwiche EA, Pestka JJ , Tortorello ML. 1985. The Veillonellae: gram-negative cocci with a

492

493

494

495

496

497

498

499 unique physiology. Annual Review of Microbiology 39:175-193

Dewhirst FE, Chen T, Izard J, Paster BJ, Tanner AC, Yu WH, Lakshmanan A, Wade

WG. 2010. The human oral microbiome. Journal of Bacteriology 192(19):5002-5017

DOI:10.1128/JB.00542-10

Diaz PI, Chalmers NI, Rickard AH, Kong C, Milburn CL, Palmer RJ, Jr., Kolenbrander

PE. 2006. Molecular characterization of subject-specific oral microflora during initial

colonization of enamel. Applied and Environmental Microbiology 72(4):2837-2848

DOI:10.1128/AEM.72.4.2837-2848.2006

500 Dotis J, Printza N, Stabouli S , Papachristou F. 2015. Kocuria species peritonitis: although

501

502

rare, we have to care. Peritoneal Dialysis International 35(1):26-30

DOI:10.3747/pdi.2013.00138

503

504

505

Du XJ, Han R, Li P , Wang S. 2015. Comparative proteomic analysis of Cronobacter sakazakii isolates with different virulences. Journal of Proteomics 128:344-351

DOI:10.1016/j.jprot.2015.08.013

506 Edgar RC. 2010. Search and clustering orders of magnitude faster than BLAST. Bioinformatics

507 26(19):2460-1. DOI: 10.1093/bioinformatics/btq461

508 Fure S, Krasse B. 1988. Comparison between different methods for sampling cariogenic

509 microorganisms in persons with exposed root surfaces. Oral Microbiology and Immunology

510 3(4):173-176

511 Gao W, Weng J, Gao Y, Chen X. 2013. Comparison of the vaginal microbiota diversity of

512 women with and without human papillomavirus infection a cross-sectional study. BMC

513 Infectious Diseases 13:271 DOI:10.1186/1471-2334-13-271

514 Gravenmade EJ , Vissink A. 1992. Management of the oral features of Sjögren's syndrome.

515 Netherlands Journal of Medicine 40(3-4):117-124 
516 Hayashi Y, Saito T, Ohshima T, Nakagawa Y, Arita T, Yashima A, Makino T, Konnai R,

517 Gomi K, Arai T , Maeda N. 2014. Terminal RFLP analysis to determine the oral microbiota

518 with hyposalivation. Archives of Microbiology 196(7):489-496 DOI:10.1007/s00203-014-0987-x

519 Kawamura Y, Hou XG, Sultana F, Miura H, Ezaki T. 1995. Determination of 16S rRNA

520 sequences of Streptococcus mitis and Streptococcus gordonii and phylogenetic relationships

521 among members of the genus Streptococcus. International Journal of Systematic Bacteriology

522 45(2):406-408

523 Keijser BJ, Zaura E, Huse SM, van der Vossen JM, Schuren FH, Montijn RC, ten Cate JM

524 , Crielaard W. 2008. Pyrosequencing analysis of the oral microflora of healthy adults. Journal

525 of Dental Research 87(11):1016-1020 DOI:10.1177/154405910808701104

526 Kolavic SA, Gibson G, al-Hashimi I, Guo IY. 1997. The level of cariogenic micro-organisms

527 in patients with Sjögren's syndrome. Special Care in Dentistry 17(2):65-69

528 Leung KC, Leung WK, McMillan AS. 2007. Supra-gingival microbiota in Sjögren's

529 syndrome. Clinical Oral Investigations 11(4):415-423 DOI:10.1007/s00784-007-0132-1

530 Li M, Zou Y, Jiang Q, Jiang L, Yu Q, Ding X, Yu Y. 2016. A preliminary study of the oral

531 microbiota in Chinese patients with Sjögren's syndrome. Archives of Oral Biology 70:143-148

532 DOI:10.1016/j.archoralbio.2016.06.016

533 Lin DF, Yan SM, Zhao Y, Zhang W, Li MT, Zeng XF, Zhang FC, Dong Y. 2010. Clinical

534 and prognostic characteristics of 573 cases of primary Sjögren's syndrome. Chinese Medical

535 Journal (Engl.) 123(22):3252-3257

536 Lu H, Ren Z, Li A, Zhang H, Jiang J, Xu S, Luo Q, Zhou K, Sun X, Zheng S, Li L. 2016.

537 Deep sequencing reveals microbiota dysbiosis of tongue coat in patients with liver carcinoma.

538 Scientific Reports 6:33142 DOI:10.1038/srep33142

539 MacFarlane TW. 1984. The oral ecology of patients with severe Sjögren's syndrome. Microbios

540 41:99-106

541 Mashima I, Nakazawa F. 2014. The influence of oral Veillonella species on biofilms formed

542 by Streptococcus species. Anaerobe 28:54-61 DOI:10.1016/j.anaerobe.2014.05.003

543 Noorda WD, Purdell-Lewis DJ, van Montfort AM, Weerkamp AH. 1988. Monobacterial and

544 mixed bacterial plaques of Streptococcus mutans and Veillonella alcalescens in an artificial

545 mouth: development, metabolism, and effect on human dental enamel. Caries Research

546 22(6):342-7 DOI:10.1159/000261134

547 Pedersen AM, Bardow A, Nauntofte B. 2005. Salivary changes and dental caries as potential

548 oral markers of autoimmune salivary gland dysfunction in primary Sjögren's syndrome. $B M C$

549 Clinical Pathology 5(1):4 DOI:10.1186/1472-6890-5-4

550 Pozharitskaia MM, Morozova LV. 1988. Antibacterial protection and the microbiological

551 status of the oral cavity in Sjögren's disease. Zhurnal Mikrobiologii, Epidemiologii $i$

552 Immunobiologii (6):11-15

553 Qin B, Wang J, Yang Z, Yang M, Ma N, Huang F , Zhong R. 2015. Epidemiology of primary

554 Sjögren's syndrome: a systematic review and meta-analysis. Annals of the Rheumatic Diseases

555 74(11):1983-1989 DOI:10.1136/annrheumdis-2014-205375

556 Rogosa M. 1964. The genus Veillonella. I . General cultural, ecological, and biochemical

557 considerations. Journal of Bacteriology 87:162-170

558 Rosselló-Mora R, Amann R. 2001. The species concept for prokaryotes. FEMS Microbiology

559 Reviews 25(1):39-67 
560 Samaranayake LP, MacFarlane TW, Lamey PJ , Ferguson MM. 1986. A comparison of oral 561 rinse and imprint sampling techniques for the detection of yeast, coliform and Staphylococcus

562 aureus carriage in the oral cavity. Journal of Oral Pathology 15:386-388

563 Savini V, Catavitello C, Masciarelli G, Astolfi D, Balbinot A, Bianco A, Febbo F, D'Amario

564 C , D'Antonio D. 2010. Drug sensitivity and clinical impact of members of the genus Kocuria.

565

566

567

568

569

570

571

572

573

574

575

576

577

578

579

580 Journal of Medical Microbiology 59(Pt 12):1395-1402 DOI:10.1099/jmm.0.021709-0

Schloss PD, Westcott SL, Ryabin T, Hall JR, Hartmann M, Hollister EB, Lesniewski RA, Oakley BB, Parks DH, Robinson CJ, Sahl JW, Stres B, Thallinger GG, Van Horn DJ, Weber CF. 2009. Introducing mothur: open-source, platform-independent, communitysupported software for describing and comparing microbial communities. Applied and Environmental Microbiology 75(23):7537-7541 DOI:10.1128/AEM.01541-09

Schulte-Pelkum J, Fritzler M , Mahler M. 2009. Latest update on the Ro/SS-A autoantibody system. Autoimmunity Reviews 8(7):632-637 DOI:10.1016/j.autrev.2009.02.010

Scully C. 1986. Sjögren's syndrome: clinical and laboratory features, immunopathogenesis, and management. Oral Surgery, Oral Medicine, Oral Pathology 62(5):510-523

Segata N, Izard J, Waldron L, Gevers D, Miropolsky L, Garrett WS, Huttenhower C. 2011. Metagenomic biomarker discovery and explanation. Genome Biology 12(6):R60. DOI:10.1186/gb-2011-12-6-r60

Siddiqui H, Chen T, Aliko A, Mydel PM, Jonsson R, Olsen I. 2016. Microbiological and bioinformatics analysis of primary Sjögren's syndrome patients with normal salivation. Journal of Oral Microbiology 8:31119 DOI:10.3402/jom.v8.31119

581 Szymula A, Rosenthal J, Szczerba BM, Bagavant H, Fu SM, Deshmukh US. 2014. T cell

582

583

584

585

586

587

588

589 epitope mimicry between Sjögren's syndrome Antigen A (SSA)/Ro60 and oral, gut, skin and vaginal bacteria. Clinical Immunology 152(1-2):1-9 DOI:10.1016/j.clim.2014.02.004

Takahashi N, Nyvad B. 2011. The role of bacteria in the caries process: ecological perspectives. Journal of Dental Research 90(3):294-303 DOI:10.1177/0022034510379602 Tennert C, Fuhrmann M, Wittmer A, Karygianni L, Altenburger MJ, Pelz K, Hellwig E, Al-Ahmad A. 2014. New bacterial composition in primary and persistent/secondary endodontic infections with respect to clinical and radiographic findings. Journal of Endodontics 40(5):670677 DOI:10.1016/j.joen.2013.10.005

590

591

592

593 van der Meulen TA, Harmsen H, Bootsma H, Spijkervet F, Kroese F, Vissink A. 2016. The microbiome-systemic diseases connection. Oral Diseases 22(8):719-734 DOI:10.1111/odi.12472 van't Hof W, Veerman EC, Nieuw Amerongen AV , Ligtenberg AJ. 2014. Antimicrobial defense systems in saliva. Monographs in Oral Science 24:40-51 DOI:10.1159/000358783 Vitali C, Bombardieri S, Jonsson R, Moutsopoulos HM, Alexander EL, Carsons SE. 2002. Classification criteria for Sjögren's syndrome: a revised version of the European criteria proposed by the American-European Consensus Group. Annals of the Rheumatic Diseases 61(6):554-558

598

599

600 Wescombe PA, Hale JD, Heng NC, Tagg JR. 2012. Developing oral probiotics from Streptococcus salivarius. Future Microbiology 7(12):1355-1371 DOI:10.2217/fmb.12.113

601

602

603

604 Xiao C, Ran S, Huang Z , Liang J. 2016. Bacterial Diversity and Community Structure of Supragingival Plaques in Adults with Dental Health or Caries Revealed by 16S Pyrosequencing. Frontiers in Microbiology 7:1145 DOI:10.3389/fmicb.2016.01145

Ximénez-Fyvie LA, Haffajee AD, Socransky SS. 2000. Microbial composition of supra- and subgingival plaque in subjects with adult periodontitis. Journal of Clinical Periodontology

605 27(10):722-732 
606 Yang F, Zeng X, Ning K, Liu KL, Lo CC, Wang W, Chen J, Wang D, Huang R, Chang X, 607 Chain PS, Xie G, Ling J, Xu J. 2012. Saliva microbiomes distinguish caries-active from 608 healthy human populations. The ISME Journal 6(1):1-10 DOI:10.1038/ismej.2011.71

609 Zaura E, Keijser BJ, Huse SM , Crielaard W. 2009. Defining the healthy "core microbiome" 610 of oral microbial communities. BMC Microbiology 9:259 DOI:10.1186/1471-2180-9-259

611 Zhang X, Zhang D, Jia H, Feng Q, Wang D, Liang D, Wu X, Li J, Tang L, Li Y, Lan Z, 612 Chen B, Li Y, Zhong H, Xie H, Jie Z, Chen W, Tang S, Xu X, Wang X, Cai X, Liu S, Xia Y, 613 Li J, Qiao X, Al-Aama JY, Chen H, Wang L, Wu QJ, Zhang F, Zheng W, Li Y, Zhang M, 614 Luo G, Xue W, Xiao L, Li J, Chen W, Xu X, Yin Y, Yang H, Wang J, Kristiansen K, Liu L, 615 Li T, Huang Q, Li Y , Wang J. 2015. The oral and gut microbiomes are perturbed in 616 rheumatoid arthritis and partly normalized after treatment. Nature Medicine 21(8):895-905

617 DOI:10.1038/nm.3914

618 Zhang Z, Deng W, Wang S, Xu L, Yan L, Liao P. 2017. First case report of infection caused 619 by Cupriavidus gilardii in a non-immunocompromised Chinese patient. IDCases 10:127-129 620 DOI:10.1016/j.idcr.2017.10.009 


\section{Figure 1 (on next page)}

Heatmap that all samples were clustered based on the microbial community similarity. The genera with relative abundance more than $1 \%$ are listed in the column and clustered according to the phylogeny.

PR: rinse samples of pSS patients;

CR: rinse samples of healthy controls. 

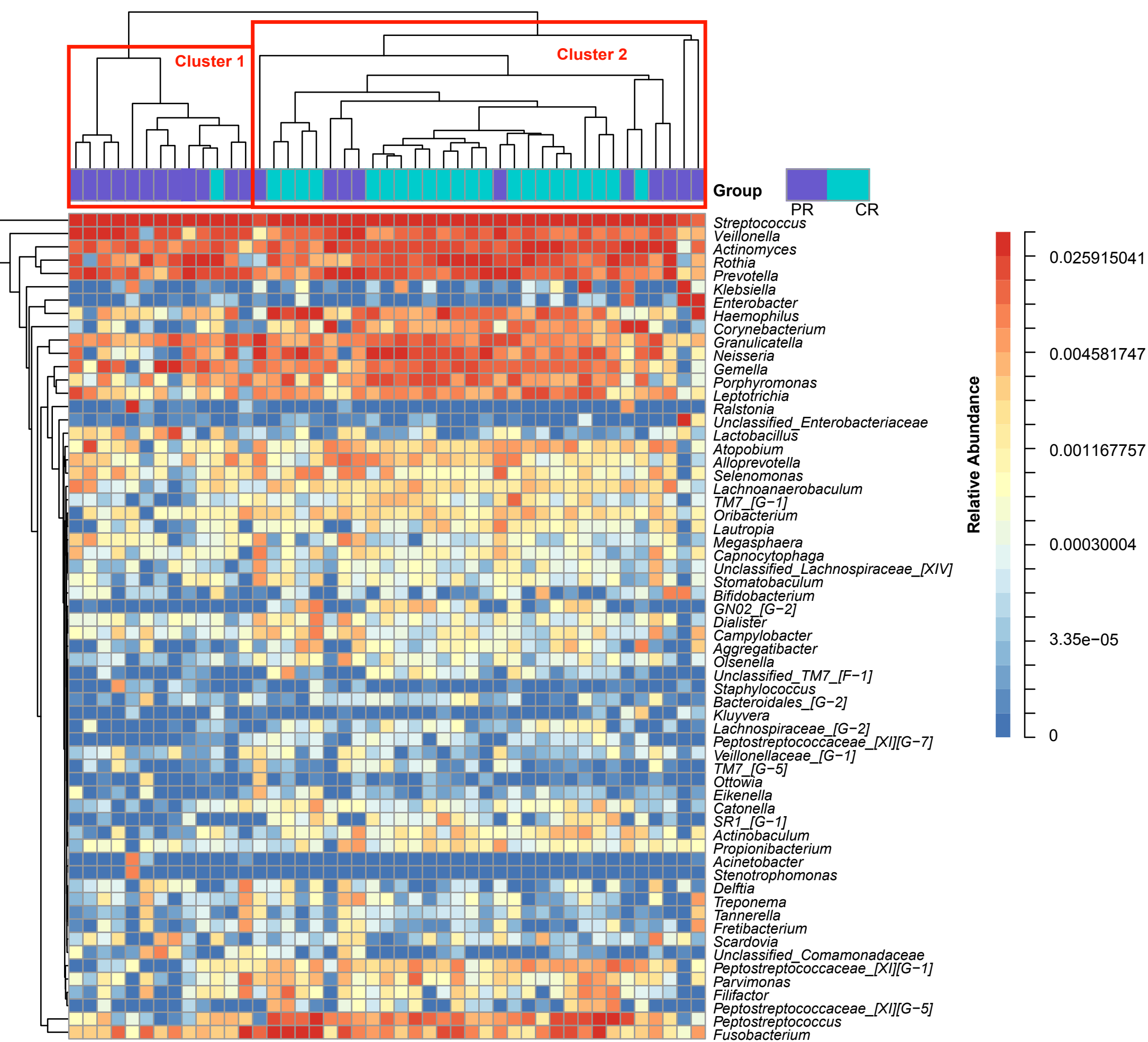

Peptostreptococcaceae_[XI][G-7]

Veillonellaceae_[G-1]

TM7 [G-5]

Ottowia

Eikenella

Catonella

SR1_[G-1]

Actinobaculum

Acinetobacter

Acinetobacter
Stenotrophomonas

Delftia

Treponema

Fretibacterium

Scardovia

Unclassified Comamonadaceae

Peptostreptococcaceae_[XI][G-1]

Parvimonas

Peptostreptococcaceae [XI][G-5]

Peptostreptococcus
Fusobacterium 


\section{Figure 2 (on next page)}

Comparisons of alpha and beta diversity indices between primary Sjögren's syndrome (pSS) patients and healthy controls.

Higher Ace (A), Chaol (B), Pielou (C) and Shannon (D) indices correlate with higher alpha diversity, while higher Simpson index (E) correlates with lower alpha diversity. (F) Comparison of principal coordinates analysis (PCOA) of primary Sjögren's syndrome (pSS) patients and healthy controls. Each dot represents one sample and the distance between the samples represents the difference in community composition of the samples.

** $p<0.01$

PR: rinse samples of $\mathrm{pSS}$ patients;

CR: rinse samples of healthy controls. 

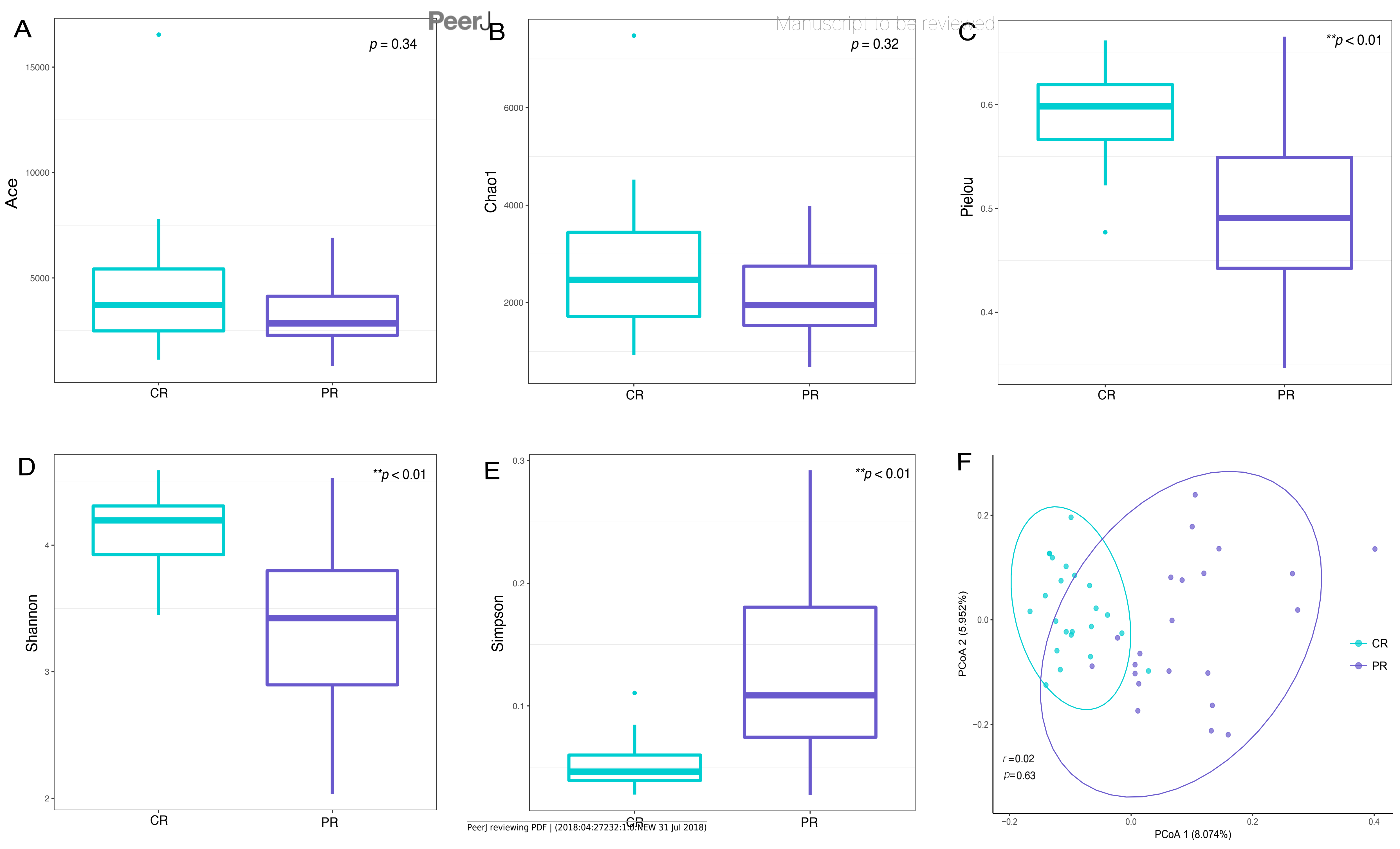


\section{Figure 3 (on next page)}

Comparison of the genera with LDA score $>4$ (highlighted by the red box) which calculated by LEfSe analysis between primary Sjögren's syndrome (pSS) patients and healthy controls.

PR: rinse samples of $\mathrm{pSS}$ patients;

CR: rinse samples of healthy controls. 


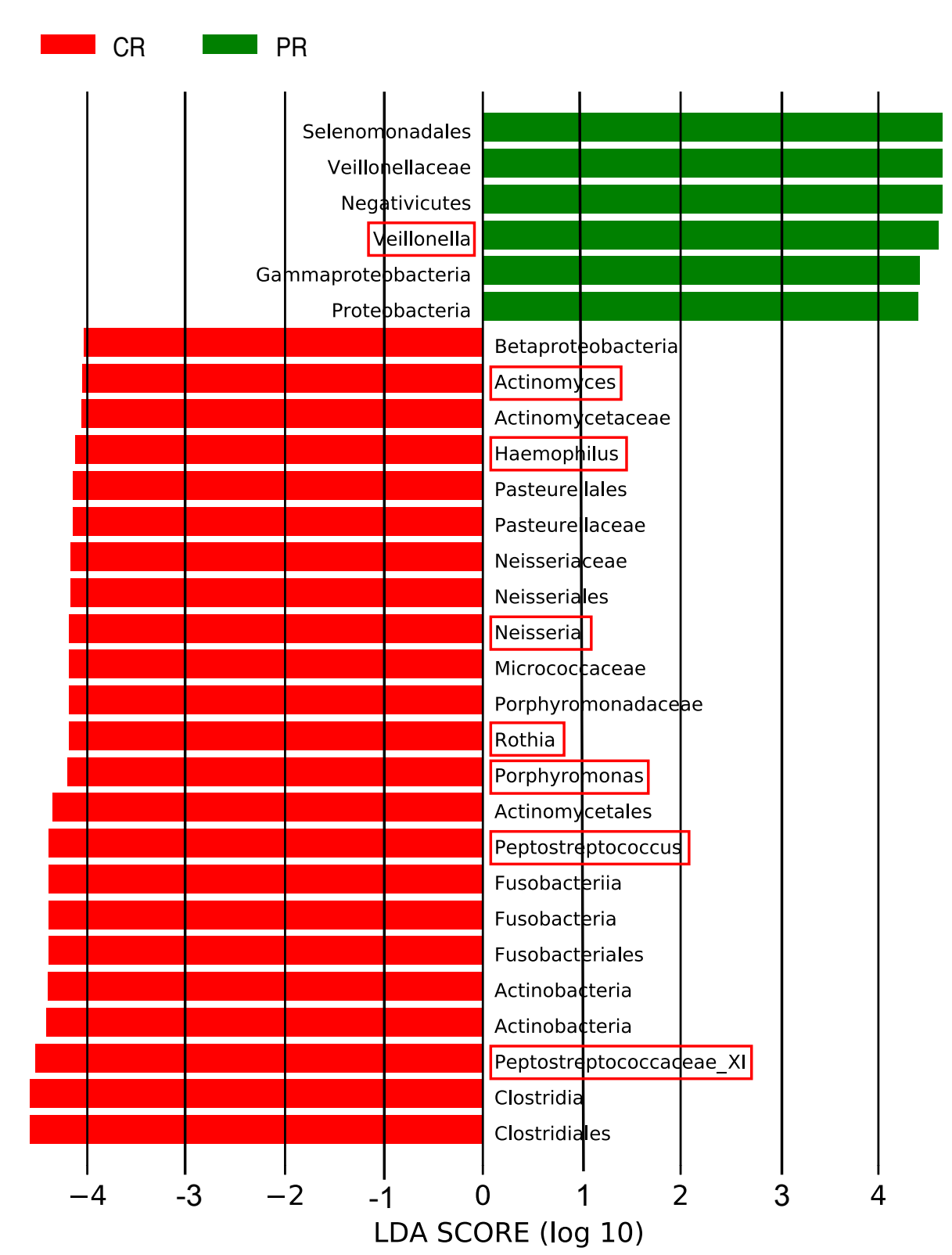




\section{Figure 4 (on next page)}

\section{Venn diagram.}

$A$ : the genera shared by two groups; $B$ : the genera shared by $C R$ except the genera in $A ; A+B$ : the genera shared by one group; $D$ : the unique genera of one group; $C$ : the genera of one group which belonged to none of $A, B$ and $D$.

PR: rinse samples of pSS patients;

CR: rinse samples of healthy controls. 


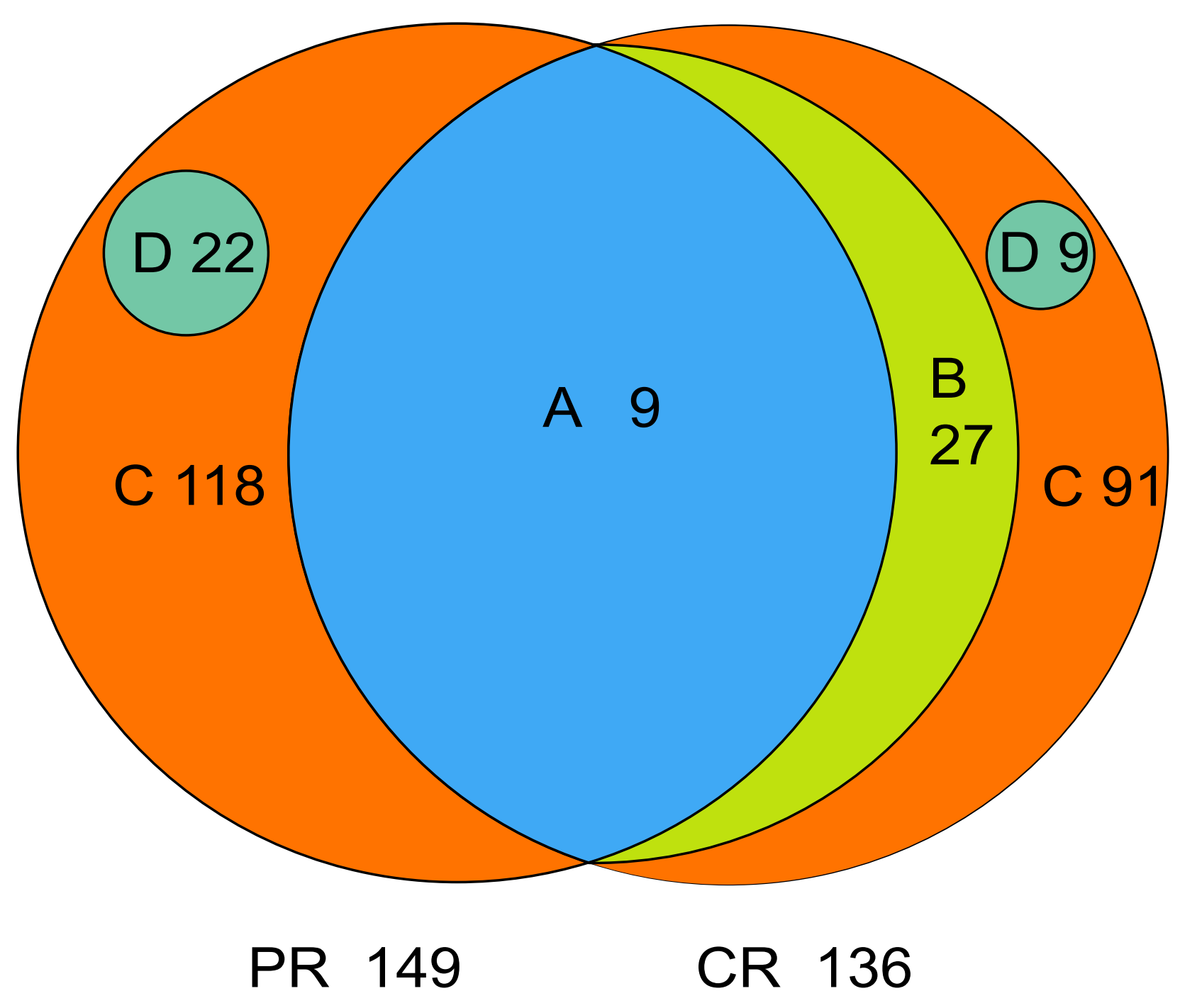




\section{Table $\mathbf{1}$ (on next page)}

Demographic and clinical data of primary Sjögren's syndrome (pSS) patients and healthy controls

a independent-samples t-tests

${ }^{\mathrm{b}}$ chi-square test

** $p<0.01$ 
1 Table 1. Demographic and clinical data of primary Sjögren's syndrome (pSS) patients and

2 healthy controls

\begin{tabular}{|c|c|c|c|}
\hline Characteristics & Patients $(n=22)$ & Healthy controls $(n=23)$ & $p$ value \\
\hline Gender (M/F) & $0 / 22$ & $0 / 23$ & \\
\hline $\operatorname{Age}^{\mathrm{a}}($ Mean $\pm \mathrm{SD}$, years $)$ & $61.50 \pm 7.47$ & $58.00 \pm 5.56$ & 0.08 \\
\hline $\begin{array}{l}\text { No. of individuals with } \\
\text { oral mucosal lesion during } \\
\text { sampling }\end{array}$ & 0 & 0 & \\
\hline $\begin{array}{l}\text { No. of individuals with any } \\
\text { other systemic diseases }\end{array}$ & 0 & 0 & \\
\hline Radiotherapy & 0 & 0 & \\
\hline $\begin{array}{l}\text { No. of individuals taking } \\
\text { drugs with side effects of } \\
\text { dry mouth for } 3 \text { months }\end{array}$ & 0 & 0 & \\
\hline $\operatorname{DMFT}^{\mathrm{a}, * *}(\mathrm{Mean} \pm \mathrm{SD})$ & $22.95 \pm 6.69$ & $8.74 \pm 5.21$ & $<0.01$ \\
\hline $\mathrm{DMFS}^{\mathrm{a}, * *}(\mathrm{Mean} \pm \mathrm{SD})$ & $74.64 \pm 30.52$ & $29.04 \pm 17.74$ & $<0.01$ \\
\hline Prevalence of caries ${ }^{b}(\%)$ & 95.45 & 69.57 & 0.06 \\
\hline
\end{tabular}

3 a independent-samples t-tests

$4 \mathrm{~b}^{\mathrm{b}}$ chi-square test

$5 * * p<0.01$ 


\section{Table 2 (on next page)}

Relative abundance of core microbiome and unique genera in primary Sjögren's syndrome (pSS) patients and healthy controls

A: the genera shared by two groups; $B$ : the genera shared by $C R$ except the genera in A; $A+B$ : the genera shared by one group; $D$ : the unique genera of one group; $C$ : the genera of one group which belonged to none of $A, B$ and $D$. 
1 Table 2. Relative abundance of core microbiome and unique genera in primary Sjögren's

2 syndrome (pSS) patients and healthy controls

\begin{tabular}{llll}
\hline \multicolumn{2}{l}{ Classification } & Genera & $\begin{array}{l}\text { Relative abundance } \\
(\text { Mean } \pm \text { SD, } \%)\end{array}$ \\
\hline \multirow{2}{*}{ A } & pSS patients & 9 & $71.88 \pm 23.28$ \\
& healthy controls & 9 & $67.64 \pm 10.26$ \\
\cline { 2 - 4 } A + B & pSS patients & 9 & $71.88 \pm 23.28$ \\
& healthy controls & 36 & $95.90 \pm 4.06$ \\
\cline { 2 - 4 } C & pSS patients & 118 & $26.83 \pm 23.54$ \\
& healthy controls & 91 & $4.05 \pm 4.02$ \\
\cline { 2 - 4 } D & pSS patients & 22 & $1.28 \pm 5.69$ \\
& healthy controls & 9 & $0.05 \pm 0.13$ \\
\hline
\end{tabular}

3 A: the genera shared by two groups; B: the genera shared by CR except the genera in A; A+B:

4 the genera shared by one group; D: the unique genera of one group; $C$ : the genera of one group

5 which belonged to none of $\mathrm{A}, \mathrm{B}$ and $\mathrm{D}$. 